\title{
LA LEGA NORD E LA TRANSIZIONE ITALIANA
}

di Roberto Biorcio

La Lega Nord ha avuto un ruolo fondamentale nella trasformazione del sistema politico italiano: ha contribuito in modo decisivo alla crisi dei partiti di governo della Prima repubblica, ha introdotto importanti innovazioni nella politica italiana e ha sinora ostacolato il consolidamento di un sistema politico bipolare. Se la transizione italiana appare lunga, complessa e ancora largamente incompiuta (Pasquino 1995, VI), il contributo del partito diretto da Umberto Bossi è già oggi chiaramente identificabile.

I successi elettorali leghisti hanno aperto la crisi dei partiti di governo, avviando il processo di transizione a un nuovo sistema politico. Il nuovo soggetto politico ha messo in discussione il linguaggio, i temi, le polarizzazioni che caratterizzavano la vita politica italiana dal secondo dopoguerra. Ha politicizzato questioni e problemi che in molti paesi europei sono stati gestiti $\mathrm{da}$ formazioni etno-nazionaliste, neo-populiste e neo-liberiste. $\mathrm{Ha}$ fornito così un contributo rilevante alla trasformazione della cultura politica della Prima repubblica, influenzando in particolare le formazioni politiche di centro-destra che hanno vinto le elezioni politiche del 1994.

La Lega Nord ha costruito in pochi anni uno dei più importanti attori politici italiani, in grado di condizionare profondamente la vita politica nelle regioni dell'Italia settentrionale. Se nelle regioni del Centro e del Sud si è affermata una competizione tendenzialmente bipolare, fra centro-destra e centro-sinistra, nelle più importati regioni del Nord la Lega ha creato un

Questo articolo rappresenta una rielaborazione del paper presentato al Workshop ECPR Italy. Changes, Constraints \& Choice, diretto da Leonardo Morlino e David Hine, Università di Warwick (Uk), 23-28 marzo 1998.

RIVISTA ITALIANA DI SCIENZA POLITICA / a. XXIX, n. 1, aprile 1999 
terzo polo incompatibile con i precedenti, che cerca di sfruttare ogni opportunità per alimentare la protesta popolare.

Nella prima sezione di questo lavoro analizzeremo lo stretto intreccio fra $\mathrm{i}$ passaggi della crisi italiana e l'iniziativa politica leghista. Cercheremo poi di mettere a fuoco le innovazioni politiche introdotte dalla Lega e la loro influenza duratura sulla politica italiana: il ruolo assunto dalla questione settentrionale, il rilievo acquisito dalle appartenenze territoriali, la politicizzazione della protesta fiscale. Prenderemo quindi in esame i tratti della cultura politica e la caratteristiche organizzative che rendono il nuovo soggetto politico molto diverso da tutti gli altri partiti, e difficilmente integrabile nelle coalizioni che competono per il governo nazionale. Infine, analizzeremo le trasformazioni del mercato elettorale nelle regioni del Nord e i loro effetti sul sistema politico italiano.

\section{Il voto per la Lega e la crisi della Prima repubblica}

Negli anni Ottanta si erano formate in Veneto, Piemonte e Lombardia embrionali leghe autonomiste che avevano svolto un ruolo del tutto marginale nel sistema politico italiano. Queste formazioni avevano strutture organizzative quasi inesistenti, prive di risorse e di militanti, spesso in difficoltà a raccogliere le firme per presentarsi alle elezioni. Per la scarsità di risorse organizzative proprie $\mathrm{i}$ primi nuclei autonomisti hanno soprattutto sfruttato le opportunità offerte delle diverse scadenze elettorali per attirare l'attenzione del pubblico. L'appello all'identità etnica e la difesa degli interessi specifici delle regioni dell'Italia settentrionale hanno però raccolto consensi molto limitati e fluttuanti. Il tentativo di mobilitare la protesta regionalista sembrava destinato al fallimento.

Nel maggio 1989 la conquista di quasi mezzo milione di voti in Lombardia ha aperto la fase dei ripetuti successi elettorali leghisti che hanno destabilizzato il sistema dei partiti della Prima repubblica ${ }^{1}$. La Lega Lombarda sotto la direzione di Bossi ha trasformato la protesta regionalista in battaglia generale contro la partitocrazia romana. L'obiettivo dell'autonomia di

1 Sull'improvviso e imprevisto successo elettorale della Lega Lombarda si vedano gli studi nel volume curato da Mannheimer (1991). 
TAB. 1. Percentuali di voto per la Lega nelle regioni settentrionali e in Italia alle elezioni politiche nazionali ed europee

\begin{tabular}{|c|c|c|c|c|c|c|c|c|c|}
\hline & \multicolumn{5}{|c|}{ Leghe regionali* } & \multicolumn{4}{|c|}{ Lega Nord } \\
\hline & $\begin{array}{c}1979 \\
(\mathrm{E})\end{array}$ & $\begin{array}{c}1983 \\
\text { (C) }\end{array}$ & $\begin{array}{c}1984 \\
(\mathrm{E})\end{array}$ & $\begin{array}{c}1987 \\
\text { (C) }\end{array}$ & $\begin{array}{c}1989 \\
(\mathrm{E})\end{array}$ & $\begin{array}{c}1992 \\
\text { (C) }\end{array}$ & $\begin{array}{c}1994 \\
\text { (C) }\end{array}$ & $\begin{array}{c}1994 \\
\text { (E) }\end{array}$ & $\begin{array}{c}1996 \\
\text { (C) }\end{array}$ \\
\hline Regioni Nord & 0,7 & 1,1 & 0,8 & 2,9 & 3,7 & 17,3 & 17,0 & 12,7 & 20,5 \\
\hline Italia & 0,5 & 0,6 & 0,5 & 1,6 & 1,8 & 8,6 & 8,4 & 6,6 & 10,1 \\
\hline
\end{tabular}

* Tavolta insieme ad altre piccole formazioni.

(C) Elezioni per la Camera dei deputati (parte proporzionale).

(E) Elezioni europee.

tutte le regioni dell'Italia settentrionale è stato presentato come la via più radicale per liquidare il potere dei partiti tradizionali e della burocrazia statale. L'etno-regionalismo delle origini è stato trasformato in una forma di populismo regionalista ${ }^{2}$.

I successi della Lega Lombarda hanno creato le basi per la formazione della Lega Nord ${ }^{3}$. Nel 1992 il Carroccio è riuscito ad ottenere il $17,3 \%$ dei voti nell'Italia settentrionale, espandendo il proprio bacino elettorale dalla Lombardia a tutte le regioni del Nord ${ }^{4}$. Il grosso dell'elettorato leghista proveniva dai partiti di governo (dalla Dc e dal Psi soprattutto). Ma il Carroccio è riuscito a conquistare anche molti elettori dei partiti di opposizione (Corbetta 1993, 250).

L'elettorato leghista nel 1992 presentava un profilo interclassista, con una penetrazione particolarmente accentuata nei settori della piccola borghesia tradizionale (commercianti, artigiani e agricoltori indipendenti) (Mannheimer 1993, 256) (tab. 2). In termini di composizione sociale, il profilo degli elettori leghisti appariva molto simile a quello tradizionale democristiano. E d'altra parte, la penetrazione più elevata del nuovo partito, non a caso, si è verificata nelle aree di antica egemonia del partito cattolico. Ma gli elettori leghisti sono spesso i più lonta-

2 Sul populismo della Lega si veda Biorcio (1991), Kitschelt (1993), Diani (1996) e Confalonieri (1997). Mario Diani ha mostrato analiticamente come nella comunicazione politica leghista si ritrovino tutti i tratti distintivi del discorso populista.

3 La Lega Nord è stata costituita formalmente il 4 dicembre 1989 , con una atto siglato da rappresentanti delle leghe della Lombardia, del Veneto, del Piemonte, della Liguria, dell'Emilia Romagna e della Toscana (Biorcio 1997, 56-59)

4 La quota dei voti raccolti dalla Lega in Lombardia si riduce dal $69 \%$ del 1990 al $44 \%$ del 1992. 
ТАВ. 2. Intenzioni di voto per la Lega nell'ambito di diversi settori sociali

\begin{tabular}{|c|c|c|c|c|c|c|c|c|}
\hline & \multicolumn{2}{|c|}{1991} & \multicolumn{2}{|c|}{1994} & \multicolumn{2}{|c|}{1996} & \multicolumn{2}{|l|}{1998} \\
\hline & Voti \% & $\mathrm{N}$ & Voti \% & $\mathrm{N}$ & Voti \% & $\mathrm{N}$ & Voti \% & $\mathrm{N}$ \\
\hline Imprend.-prof.-dirig. & 14,0 & 50 & 15,8 & 202 & 12,4 & 88 & 16,8 & 129 \\
\hline Insegnanti-impiegati & 13,3 & 249 & 16,0 & 675 & 18,8 & 609 & 21,7 & 817 \\
\hline Commerc.-artig.-agric. & 24,0 & 96 & 26,5 & 260 & 23,9 & 94 & 31,3 & 109 \\
\hline Operai-braccianti & 16,6 & 157 & 21,4 & 393 & 31,2 & 290 & 38,5 & 376 \\
\hline Casalinghe & 10,7 & 168 & 14,2 & 592 & 21,6 & 370 & 16,1 & 283 \\
\hline Pensionati & 10,7 & 234 & 15,1 & 764 & 13,8 & 429 & 14,4 & 644 \\
\hline Studenti & 14,6 & 158 & 16,0 & 224 & 18,2 & 132 & 16,6 & 293 \\
\hline Disoccupati & 21,4 & 28 & 17,0 & 83 & 21,0 & 52 & 12,5 & 91 \\
\hline Totale Nord & 14,1 & 1.140 & 16,9 & 3.233 & 19,8 & 2.099 & 22,0 & 2.784 \\
\hline
\end{tabular}
nali.

Fonte: Sondaggi Eurisko (1991) e Abacus (1994; 1996; 1998) - Regioni settentrio-

ni dalle pratiche religiose nelle tradizionali province bianche, $\mathrm{e}$ rivelano il processo di secolarizzazione accelerata che ha investito queste aree.

La domanda di cambiamento del sistema politico non è stata raccolta solo dalla Lega. Il movimento referendario guidato da Mario Segni si è fortemente impegnato su questo terreno ed è riuscito a cogliere una prima inattesa vittoria nel 1991 con il referendum sulla preferenza unica e un'affermazione decisiva il 18 aprile 1993 con il referendum per la trasformazione in senso maggioritario della legge elettorale per il Senato. Nelle regioni dell'Italia settentrionale i risultati del referendum sulla riforma elettorale hanno messo in evidenza una domanda di cambiamento del sistema di rappresentanza molto più elevata rispetto alle regioni meridionalis.

La lotta alla partitocrazia è obiettivo comune alla Lega e al movimento referendario. Ma radicalmente diverse e sostanzialmente inconciliabili sono le culture e le prospettive politiche dei due attori politici (Ricolfi 1995, 270-271). Per il movimento referendario il superamento delle degenerazioni della Prima repubblica è affidato al semplice cambiamento delle regole. Per la Lega la lotta alla partitocrazia fa leva sulla difesa di concreti in-

5 I voti favorevoli al referendum del 18 aprile 1993 sono risultati superiori ai due terzi degli elettori in tutte le regioni del Nord. Nelle regioni del Sud meno della metà degli elettori ha approvato la proposta di modifica del sistema elettorale in senso maggioritario (Corbetta e Parisi 1994, 12). 
teressi, gestiti in chiave populista, e sulla costruzione di un riferimento identitario alternativo a quelli politici tradizionali.

L'introduzione del sistema maggioritario ha avuto grande importanza per la trasformazione del sistema politico italiano, ma non ha garantito il successo elettorale alla formazione guidata da Mario Segni. La Lega ha invece saputo tradurre in consenso elettorale la crescente protesta dell'opinione pubblica molto più efficacemente di tutte le formazioni concorrenti perché ha unito la lotta alla partitocrazia alla difesa di interessi e valori diffusi nelle popolazioni del Nord ${ }^{6}$.

Nel 1993, mentre cresceva il rilievo delle inchieste della magistratura che coinvolgevano i leader dei partiti di governo, $\mathrm{i}$ consensi per il partito di Bossi sono ancora cresciuti nelle regioni del Nord, raggiungendo secondo molti sondaggi quasi il 50\% delle intenzioni di voto. Nelle elezioni amministrative, che si sono svolte secondo le nuove regole elettorali, la Lega è riuscita a conquistare da sola la maggioranza in molti consigli comunali e provinciali del Nord. Le stesse elezioni amministrative hanno reso visibile il crollo di consensi della Democrazia cristiana e del Partito socialista, indeboliti e delegittimati dalle inchieste della magistratura. In tutte le grandi città $i$ candidati dei due principali partiti di governo sono stati esclusi dal ballottaggio.

La crescita impetuosa dei voti per la Lega, le inchieste della magistratura e la trasformazione delle regole elettorali, sovrapponendo e intrecciando $\mathrm{i}$ loro effetti, hanno fatto così precipitare la crisi definitiva della Prima repubblica.

Svolte strategiche e mutamenti della base elettorale leghista

Se le affermazioni elettorali leghiste hanno destabilizzato il nostro sistema politico, la Lega si è a sua volta trasformata per fare fronte ai problemi e utilizzare le opportunità emergenti nella transizione italiana. Le svolte strategiche del nuovo partito hanno determinato rilevanti mutamenti nel profilo della sua base elettorale.

Nel 1994 la «discesa in campo» di Berlusconi e la formazione di Forza Italia hanno modificato profondamente il quadro in

6 Oltre al movimento referendario promosso da Mario Segni, si sono impegnati, in forme diverse, nella battaglia contro i partiti tradizionali il Partito Radicale di Marco Pannella e la Rete di Leoluca Orlando. 
cui si era sviluppata la prima crescita elettorale leghista. Il Carroccio, dopo un'iniziale esitazione, si è alleato con la coalizione di centro-destra e ha contribuito in modo decisivo alla sua vittoria elettorale del 27 marzo 1994. Gli alleati hanno però conteso lo spazio politico al partito di Bossi, che ha perso consensi elettorali e ha subito un netto arretramento nelle elezioni europee del 1994, soprattutto a vantaggio di Forza Italia.

La Lega era cresciuta troppo in fretta nei primi anni Novanta per avere la possibilità di consolidare l'identità politica e il proprio radicamento sociale. Aveva perciò incontrato grandi difficoltà quando aveva stretto alleanze politiche con il Polo e aveva assunto responsabilità di governo. Per salvare il partito Bossi ha deciso la rottura con Berlusconi, l'appoggio al governo Dini e la radicalizzazione degli elementi distintivi della propria identità. Viene abbandonato il federalismo a vantaggio dell'indipendentismo. Tra il 1995 e il 1997 sono state perciò investite grandi risorse sul progetto della Padania per ottenere un consolidamento di lungo termine dell'identità e dell'organizzazione. L'elettorato leghista ha assunto progressivamente tratti più definiti che, come vedremo tra poco, lo differenziano da quello dei principali partiti italiani. Nel febbraio 1997 il terzo congresso ordinario ha ratificato formalmente la nuova strategia, sostituendo la denominazione «Lega Nord - Italia Federale» con quella «Lega Nord per l'indipendenza della Padania».

La svolta politica è stata premiata dagli elettori: nel 1996 la Lega è diventata il primo partito nelle regioni dell'Italia settentrionale con il $20,5 \%$ dei voti. La rottura con il centro-destra e la svolta indipendentista hanno modificato la fisionomia sociale dell'elettorato leghista, rendendolo più popolare (tab. 2). Il partito ha esteso i propri consensi soprattutto fra gli operai dell'Italia settentrionale. All'inizio degli anni Novanta solo un operaio su sei votava per il partito di Bossi; nel 1996 ha votato per la Lega quasi un terzo degli operai residenti nelle regioni dell'Italia settentrionale. E d'altra parte, la maggiore espansione del voto leghista si registra nei collegi elettorali con il più elevato tasso di industrializzazione, soprattutto nelle aree in cui prevale la piccola industria (Diamanti 1996, 22). Uno sviluppo complementare ha invece avuto l'elettorato di Forza Italia nelle regioni del Nord. Il partito di Berlusconi ha perduto consensi fra gli operai e ha invece aumentato i voti nell'ambito dei ceti superiori e della piccola borghesia. Il profilo sociale degli elettori della Lega è diventato molto simile a quello dei partiti neo- 
populisti europei. I successi elettorali di queste formazioni politiche hanno modificato ovunque la loro base sociale di riferimento: da un radicamento iniziale nella piccola borghesia a una crescente influenza fra gli operai (Taggart 1996).

La radicalizzazione della posizione indipendentista utile per consolidare l'identità e rafforzare l'organizzazione ha creato un relativo isolamento della Lega, riducendo il suo peso nella politica italiana. Si sono accentuate tensioni fra le diverse componenti del movimento, in particolare fra il gruppo dirigente lombardo e una parte dei quadri veneti.

Nell'ultima fase il Carroccio ha cercato di riprendere l'iniziativa politica, con una nuova svolta strategica che è stata sancita da due congressi straordinari. Il congresso di Milano, nel marzo 1998, ha lasciato cadere la secessione come prospettiva immediata e ha delineato un percorso graduale per realizzare gli obiettivi strategici del movimento. Mantenendo ferma la prospettiva indipendentista, la Lega ha assunto come riferimento l'esperienza della Scozia, che ha ottenuto il riconoscimento del diritto all'elezione di un parlamento autonomo, e potrà gestire una serie di poteri delegati dal parlamento britannico (devolution).

Questo progetto ha incontrato però difficoltà esterne e non ha risolto le divergenze interne. La costruzione di un'alleanza fra Polo e Lega è stata sperimentata in Veneto ${ }^{7}$. Questa esperienza, duramente sconfessata da Bossi, ha provocato la rottura con il gruppo dirigente veneto, che è uscito dal partito e ha fondato la Liga Veneta Repubblica. La sviluppo della prospettiva rigorosamente regionalista del gruppo veneto permetterebbe un riassorbimento del fenomeno leghista nel contesto di una competizione bipolare aperta ad alleanze con marginali formazioni localiste.

Bossi combatte questo progetto e propone una diversa strategia per inserirsi nelle contraddizioni della politica romana mantenendo l'unità della Lega come rappresentante di tutto il Nord. Le possibilità di accordi elettorali a livello locale con Polo e Ulivo sono rifiutate per evitare spinte alla disgregazione del movimento. Il congresso straordinario di Brescia nell'ottobre 1998 ha proposto la strategia della «Lega di governo» per acquistare peso nella politica nazionale: la disponibilità del par-

7 Un documento leghista per l'autodeterminazione del popolo veneto è stato votato da Forza Italia nel consiglio regionale, mentre la Lega ha appoggiato la proposta avanzata dal Polo per ottenere lo statuto di regione autonoma. 
tito ad appoggiare un futuro governo nazionale, per ottenere riconoscimenti politici e avviare un processo che accresca, in qualche forma istituzionale, l'autonomia delle regioni dell'Italia settentrionale. Per competere con i due principali schieramenti politici nelle regioni del Nord si progetta di diversificare l'offerta politica con la costruzione del Blocco padano: un'alleanza della Lega con le formazioni promosse per rappresentare le diverse componenti della «società padana» (cattolici, agricoltori, pensionati, imprenditori), con liste civiche locali e movimenti regionalisti.

\section{La questione settentrionale}

La Lega ha posto al centro della propria battaglia politica la frattura Nord/Sud e ha avuto un indubbio successo: l'interesse per la «questione meridionale» si è ridotto, mentre si moltiplicano i dibattiti e i convegni sulla «questione settentrionale», che ha sollecitato sempre più l'attenzione di tutte le forze politiche italiane.

La frattura Nord/Sud è sempre stata profonda sul piano economico dalla costituzione dello stato italiano, ma l'assenza di forti differenze etnoculturali aveva reso difficile la mobilitazione territoriale (Trigilia 1994, 82). Nel secondo dopoguerra il divario economico Nord/Sud si è ridotto ma non si è sviluppato nelle regioni meridionali un sistema produttivo autonomo. Anche se non è facile una stima esatta, il Nord ha finanziato in misura preponderante il bilancio della stato, mentre il Sud ha ricevuto risorse molto superiori a quelle prodotte (Becchi 1995, 495). Negli anni Ottanta questo trasferimento di risorse ha contribuito fortemente alla crescita del debito pubblico, che ha provocato l'aumento della pressione fiscale. Il parlamento ha cancellato fra il 1991 e il 1992 le misure di intervento straordinario per il Mezzogiorno, per evitare il referendum che ne chiedeva l'abrogazione. La protesta per la politica economica del governo era facilmente focalizzabile contro la redistribuzione territoriale delle risorse. La Lega ha saputo cogliere questa opportunità e si è presentata come portavoce degli interessi e dei valori delle regioni settentrionali. Il Carroccio ha cercato di reinterpretare in chiave antimeridionalista l'antipatia di molti cittadini verso i pubblici dipendenti: i funzionari dell'amministrazione, gli insegnanti, $i$ giudici. Il nuovo partito ha condotto campagne politi- 
che permanenti contro la «scuola coloniale», contro la «giustizia coloniale» e contro episodi di assenteismo e inefficienza di pubblici dipendenti, sospettati di essere di origine meridionale. La Lega ha rivendicato il diritto prioritario dei cittadini dell'Italia settentrionale a coprire, nei rispettivi ambiti regionali, i ruoli nella pubblica amministrazione e nell'insegnamento.

La questione meridionale è stata così ribaltata facendo emergere la necessità di difendere non solo gli interessi, ma la stessa identità etno-culturale delle regioni dell'Italia del Nord «colonizzate e oppresse» da istituzioni dominate da un ceto politico e da un'etnia estranea. Nessuna forza politica italiana aveva mai gestito i sentimenti di ostilità nei confronti degli immigrati meridionali. I successi elettorali leghisti hanno legittimato gli atteggiamenti antimeridionalisti nelle regioni del Nord, soprattutto fra i ceti popolari (commercianti, artigiani, e operai) e in generale fra i cittadini più anziani e meno istruiti.

La politicizzazione della frattura Nord-Sud e l'antimeridionalismo sono serviti a fare emergere le identità regionali prima, e quella padana poi. La Lega ha seguito la classica strategia comunicativa dei movimenti populisti, dedicando più impegno a elaborare la figura del nemico che a valorizzare le specificità delle popolazioni dell'Italia settentrionale (Confalonieri 1997, 92).

La questione settentrionale non trova fondamento in uno specifico «malessere» socio-economico percepito dalla popolazione delle regioni del Nord. Le indagini demoscopiche mostrano che in generale chi abita in queste regioni è molto più soddisfatto delle condizioni di vita personale e familiare e dei principali servizi sociali (scuola, sanità e trasporti) degli abitanti del Mezzogiorno (Natale 1997). Solo per alcuni aspetti dell'ambiente fisico e sociale (l'inquinamento dell'aria e la microcriminalità) - ovunque percepiti come molto insoddisfacenti - il disagio delle regioni settentrionali risulta lievemente superiore (tab. 3).

La questione settentrionale si nutre soprattutto della apparente contraddizione fra benessere economico e malessere politico (Diamanti 1997, 1064). La tensione fra società civile e rappresentanza politica nelle regioni settentrionali è stata evidenziata dai livelli di partecipazione e dai risultati dei referendum del 1991 e del 1993 finalizzati alla trasformazione del sistema elettorale tradizionale.

La Lega ha proposto un diverso sbocco per questa tensione con il progetto di autogoverno del Nord - «comandiamo a casa nostra» - come radicale risoluzione dei rapporti con la classe 
TAB. 3. Soddisfazione per diversi aspetti della vita personale e dell'ambiente fisico e sociale

\begin{tabular}{lcccc}
\hline & \multicolumn{4}{c}{ Area di residenza } \\
\cline { 2 - 5 } & Nord & Centro & Sud & Italia \\
\hline Soddisfatti per: & & & & \\
Casa in cui vive & 91,2 & 93,2 & 89,7 & 91,1 \\
Zona in cui vive & 89,3 & 88,6 & 84,6 & 87,5 \\
Lavoro & 81,0 & 77,7 & 59,1 & 72,7 \\
Livello istruzione & 77,7 & 68,6 & 60,9 & 70,0 \\
Reddito familiare & 75,5 & 69,7 & 60,5 & 69,1 \\
Tenore vita & 70,4 & 71,4 & 61,5 & 67,5 \\
Investimenti & 55,6 & 52,5 & 42,0 & 50,3 \\
Scuola & 59,9 & 56,8 & 44,0 & 53,8 \\
Sanità & 64,8 & 55,7 & 33,3 & 52,0 \\
Trasporti pubblici & 56,2 & 44,2 & 35,6 & 46,6 \\
Verde & 46,8 & 46,6 & 26,8 & 39,8 \\
Traffico & 40,7 & 38,5 & 36,8 & 38,9 \\
Inquinamento aria & 32,1 & 36,0 & 41,7 & 36,3 \\
Microcriminalità & 24,7 & 32,4 & 29,7 & 28,0 \\
N. & 2.784 & 664 & 955 & 4.403 \\
\hline
\end{tabular}

Fonte: Sondaggio Abacus (1998).

politica e la burocrazia romana. L'idea che le regioni settentrionali non abbiano una adeguata rappresentanza politica è diventata molto diffusa, soprattutto fra gli operatori economici, e si sono moltiplicati i tentativi di promuovere progetti e movimenti autonomisti da parte di esponenti del centro-destra e del centro-sinistra. Il sindaco di Venezia Cacciari ha fondato il Movimento del Nord-Est, che si è presentato in alcune consultazioni amministrative. Giulio Tremonti, ex ministro del governo Berlusconi, ha promosso un movimento federalista nell'ambito del centro-destra.

I sondaggi di opinione rivelano che il tema dell'autonomia delle regioni settentrionali si è ormai affermato con forza come uno dei termini di riferimento del dibattito politico, intrecciato alle valutazioni sui suoi possibili vantaggi. L'opinione che sia necessario aumentare l'autonomia del Nord è via via cresciuta nel corso degli anni Novanta, ed è condivisa da più di due terzi dei residenti nelle regioni settentrionali (tab. 4). Nelle altre regioni la domanda di autonomia si è mantenuta a livelli molto più ridotti. 
TAB. 4. Domanda di maggiore autonomia tra Nord, Centro e Sud

\begin{tabular}{lrrrr}
\hline Area di residenza & 1991 & 1994 & 1996 & 1998 \\
\hline Nord & 54,7 & 56,2 & 62,6 & 69,0 \\
Centro & - & 36,5 & 39,5 & 39,6 \\
Sud & - & 34,5 & 43,6 & 40,0 \\
Italia & - & 44,5 & 52,1 & 53,5 \\
N. & 920 & 13.602 & 3.885 & 4.403 \\
\hline
\end{tabular}

Fonte: Sondaggi Doxa (1991) e Abacus (1994; 1996; 1998).

La politicizzazione delle appartenenze territoriali e l'identità nazionale

La configurazione delle identità territoriali degli italiani è complessa, e vede in generale la coesistenza di diversi sentimenti di appartenenza, che si sovrappongono con diversa intensità, senza escludersi (Segatti 1995, 109). Nel secondo dopoguerra sia l'appartenenza nazionale che le appartenenze subnazionali avevano avuto un ruolo limitato nell'arena politica, con l'eccezione di alcune regioni fortemente caratterizzate da autonomia linguistica e culturale (Valle d'Aosta, Sud Tirolo, Sardegna).

Questo quadro si è modificato negli anni Ottanta, quando le piccole leghe autonomiste avevano tentato di politicizzare le identità regionali, qualificandole come nazionali. La Lega Nord, per superare la condizione di semplice alleanza di formazioni regionaliste, ha cercato di costruire un riferimento identitario unico. Nel 1989, nel momento in cui si è avviata la fusione delle leghe autonomiste in un unico movimento politico, è stata per la prima volta delineata l'idea della Padania concepita come «comunità multiregionale della stessa cultura». Senza negare le singole identità regionali e l'esigenza di specifiche forme di autogoverno, si è affermata la consapevolezza che solo con l'unità delle regioni del Nord è possibile contrastare il potere del governo nazionale ed ottenere concessioni significative sul terreno dell'autonomia politica ed economica.

Il progetto di autonomia della Padania ha trovato un potente fondamento nelle ragioni dell'economia ${ }^{8}$. La propaganda le-

8 Prima che nella propaganda leghista, l'idea di una macroregione denominata «Padania» aveva trovato spazio nelle riflessioni della Fondazione Agnelli. Il potenziale produttivo delle otto regioni dell'Italia settentrionale era infatti considerato adeguato a 
ghista ha enfatizzato la produttività delle regioni del Nord, e la loro condizione di creditrici nei confronti dello stato italiano, cui destinano più risorse di quante ne ricevano. Questo quadro interpretativo, simile a quello di altri movimenti etnoregionalisti $^{9}$, ha influenzato gli atteggiamenti dell'opinione pubblica delle regioni settentrionali.

Per dare una radice etnoculturale al progetto della Padania la Lega ha riproposto il classico percorso di invenzione della tradizione: «Per "tradizione inventata" si intende un insieme di pratiche [...] dotate di una natura rituale o simbolica, che si propongono di inculcare determinati valori e norme di comportamento ripetitive nelle quali è automaticamente implicita la continuità col passato» (Hobsbawn 1983, 3). Per sostenere l'idea della nazione padana sono state rievocate prima le esperienze dei liberi comuni dell'Italia medioevale, poi le tradizioni celtiche. Il «patriottismo» della nuova nazione è stato promosso con grandi rituali di massa, dalle adunate dei simpatizzanti leghisti che si celebrano periodicamente a Pontida alle manifestazioni indipendentiste lungo il Po.

La Lega ha tentato di costituire, sul piano simbolico, le istituzioni padane e di promuovere la formazione di una società civile per la nuova nazione. Sono stati riprodotti i rituali democratici di un vero e proprio stato, organizzando un referendum e l'elezione di un «parlamento padano». Nell'ultima fase l'impegno è stato soprattutto rivolto a fare emergere la società civile padana con la promozione di iniziative culturali, economiche, sportive e ricreative. Sono state fondate associazioni in quasi tutti i settori della vita sociale e si sono avviate le prime esperienze della «scuola padana».

La propaganda e le iniziative politiche della Lega hanno trasformato la configurazione delle identità territoriali nelle regioni settentrionali. Il separatismo nordista ha riaperto la discussione sul valore e il ruolo dell'identità nazionale (Rusconi 1993).

Per studiare i livelli di identificazione rispetto a diversi ambiti territoriali abbiamo fatto ricorso al classico strumento del «termometro dei sentimenti» ${ }^{10}$. Si possono considerare rilevanti

reggere senza difficoltà la sfida delle più importanti aree europee, a condizione di essere sorretto da istituzioni autonome e appropriate, «diverse dallo stato centralista» (Pacini 1992, 6).

9 Questa impostazione si può ritrovare nei movimenti etno-regionalisti delle Fiandre, della Catalogna, dei Paesi Baschi: si veda De Winter e Türsan $(1998,158)$

10 Con questa tecnica si richiede agli intervistati di esprimere il livello di impor- 


\begin{tabular}{lcccc}
\hline & \multicolumn{4}{c}{ Area di residenza } \\
\cline { 2 - 5 } & Nord & Centro & Sud & Italia \\
\hline Si sente cittadino di: & & & & \\
Italia & 65,0 & 70,4 & 82,9 & 72,3 \\
Comune & 59,9 & 57,0 & 63,7 & 61,6 \\
Regione & 57,8 & 41,8 & 48,8 & 54,2 \\
Italia settentrionale, & 49,4 & 35,1 & 47,5 & 46,6 \\
centrale o meridionale & 2.784 & 664 & 955 & 4.403 \\
N. & & &
\end{tabular}

* L'importanza dei sentimenti di appartenenza è stata rilevata con una scala di punteggi fra 1 e 10 . Sono stati considerati importanti i sentimenti a cui è stato attribuito un valore non inferiore a 8 .

Fonte: Sondaggio Abacus (1998).

i sentimenti di appartenenza cui gli intervistati hanno attribuito un punteggio superiore al livello medio generale $(7,4)^{11}$.

Possiamo verificare così che l'identità nazionale italiana resta la più diffusa in tutte le aree del paese, condivisa dal $72,3 \%$ degli intervistati (tab. 5) ${ }^{12}$. Nell'Italia settentrionale l'identità nazionale è però relativamente meno sentita rispetto alle altre regioni. Questa differenza non può essere attribuita alla particolare forza del localismo nelle regioni settentrionali, che risulta inferiore alla media nazionale. Al Nord sono invece più diffusi, rispetto alle altre aree del paese, i sentimenti di appartenenza alla regione e anche quelli per l'area territoriale multiregionale (Italia settentrionale): gli ambiti di riferimento privilegiati dalla propaganda leghista.

tanza dell'appartenenza a ciascun ambito territoriale su una scala da 1 a 10 . Sulla tecnica del «termometro dei sentimenti», si veda Biorcio e Pagani $(1997,104)$.

11 In altre indagini si è seguita una diversa procedura per rilevare i sentimenti di appartenenza, chiedendo' semplicemente di scegliere i due ambiti territoriali a cui l'intervistato si sente più legato. Questa tecnica di rilevazione non permette di valutare l'intensità dei sentimenti: in alcuni casi, anche gli ambiti indicati al primo posto possono avere scarsa rilevanza per chi non attribuisce molta importanza ai sentimenti di appartenenza territoriale. In altri casi, alcuni ambiti che non sono scelti fra i primi due possono essere ugualmente rilevanti per gli intervistati.

12 I nostri risultati sono coerenti con quelli ottenuti in anni passati da altre ricerche condotte con tecniche diverse. Paolo Segatti aveva rilevato nel 1990 che, considerando insieme la prima e la seconda scelta, il sentimento di appartenza nazionale risultava il più diffuso, condiviso dal $68 \%$ degli intervistati (Segatti 1995, 109). Conclusioni simili si sono raggiunte considerando la quota di intervistati che si dichiaravano orgogliosi di essere italiani: 71,9\% nel 1994 (Diamanti e Segatti 1994, 17). 
TAB. 6. Tipologia di appartenenze rilevanti

\begin{tabular}{lccrr}
\hline & \multicolumn{4}{c}{ Area di residenza } \\
\cline { 2 - 5 } & Nord & Centro & Sud & Italia \\
\hline Tipo di appartenenza: & & & & \\
Solo nazionale & 11,2 & 18,0 & 13,3 & 13,2 \\
Nazionale e locale & 53,8 & 52,4 & 69,4 & 59,1 \\
Solo locale & 19,1 & 16,7 & 6,1 & 14,0 \\
Né nazionale né locale & 15,8 & 12,9 & 11,2 & 13,6 \\
N. & 2.784 & 664 & 955 & 4.403 \\
\hline
\end{tabular}

Fonte: Sondaggio Abacus (1998).

TAB. 7. Sentimenti di appartenenza, per intenzioni di voto, nelle regioni settentrionali

\begin{tabular}{lcccccc}
\hline & \multicolumn{6}{c}{ Elettori } \\
\cline { 2 - 7 } & Lega & An & Fi & Ppi & Pds & Nord Italia \\
\hline Si sentono cittadini di: & & & & & & \\
Italia settentrionale & 74,6 & 55,0 & 47,7 & 37,7 & 40,6 & 49,4 \\
Regione & 70,2 & 56,1 & 62,8 & 54,2 & 57,4 & 57,8 \\
Comune & 68,3 & 51,0 & 63,1 & 63,8 & 64,1 & 59,9 \\
Italia & 52,6 & 69,5 & 70,5 & 69,0 & 73,6 & 65,0 \\
& & & & & & \\
Tipi di appartenenza: & 1,8 & 15,4 & 8,0 & 5,4 & 9,8 & 11,2 \\
Solo nazionale & 50,8 & 54,1 & 62,5 & 63,6 & 63,8 & 53,8 \\
Nazionale e locale & 35,2 & 16,8 & 14,6 & 15,2 & 12,3 & 19,1 \\
Solo locale & 12,2 & 13,6 & 14,9 & 15,7 & 14,1 & 15,8 \\
Né nazionale né locale & & & & & & \\
& 400 & 213 & 441 & 201 & 585 & 2.784 \\
N. & & & & & &
\end{tabular}

Fonte: Sondaggio Abacus - Regioni settentrionali (1998).

I sentimenti di appartenenza alla comunità nazionale e ai diversi ambiti territoriali non sono esclusivi, ma, come è noto, si sovrappongono in modo complesso. Per valutare una potenziale tensione fra appartenenza italiana e altre appartenenze subnazionali abbiamo costruito una semplice tipologia. Abbiamo distinto quattro tipi di posizioni fondamentali. Quella di chi: $a$ ) attribuisce importanza solo all'appartenenza nazionale; $b$ ) attribuisce importanza sia all'appartenenza nazionale sia all'appartenenza ad ambiti locali o subnazionali (città, regione, Nord, Centro e Sud); c) attribuisce importanza solo ad ambiti locali o subnazionali; $d$ ) non attribuisce importanza a nessuno di questi ambiti. 
La distribuzione delle quattro posizioni conferma la prevalenza della tradizionale combinazione di sentimenti di appartenenza locali e nazionali. Questo modello, che permette di definire l'Italia una «nazione di compaesani» (Segatti 1995), si mantiene largamente dominante al Sud, ma appare indebolito nelle altre regioni (tab. 6). Nell'Italia settentrionale è cresciuta in particolare la tendenza a separare dall'appartenenza nazionale le appartenenze locali e subnazionali, le uniche riconosciute come rilevanti da quasi un quinto della popolazione.

In queste regioni si può ritrovare una chiara relazione fra identità territoriali e scelte di voto. Tra gli elettori leghisti i sentimenti di appartenenza all'Italia del Nord sono i più diffusi, seguiti da quelli per la regione (tab. 7). Oltre un terzo degli elettori leghisti riconosce solo appartenenze locali e subnazionali. Il modello tradizionale che prevede la coesistenza di appartenenze nazionali e locali resta invece largamente dominante fra gli elettori del Pds, del Ppi e di Forza Italia. L'elettorato di Alleanza nazionale infine appare più soggetto a una tendenziale polarizzazione fra chi riconosce come rilevante la sola appartenenza nazionale, e chi fa riferimento unicamente ad appartenenze locali o subnazionali.

\section{Il dinamismo della piccola impresa e la protesta fiscale}

La Lega Nord ha cercato di superare la frattura di classe e di ridefinire il conflitto sociale in chiave populista. Il nuovo partito ha identificato la piccola e media impresa come referente sociale privilegiato, e ha cercato di politicizzare la protesta fiscale. Il Carroccio ha cercato di evidenziare la contrapposizione fra gli interessi comuni ai piccoli imprenditori e ai loro dipendenti e quelli della grande industria e della grande finanza.

Il progetto di radicamento sociale della Lega ha trovato un fondamento nelle trasformazioni economiche e sociali di molte aree dell'Italia settentrionale, ove le piccole imprese si sono progressivamente emancipate dalla funzione ausiliaria rispetto alla grande industria. Il radicamento nella comunità locale ha fornito risorse fondamentali per lo sviluppo, garantendo agli operatori economici conoscenze e linguaggi condivisi, possibilità di relazioni con altre imprese, servizi e infrastrutture (Bagnasco 1997, 203). Questi processi hanno avuto un particolare sviluppo nelle regioni del Nord-Est, che si sono trasformate da pe- 
riferia industriale in uno dei settori più dinamici dell'economia italiana, in grado di coniugare alta competitività e occupazione, apertura internazionale e radicamento locale, innovazione e tradizione (Anastasia e Corò 1996). Il dinamismo economico delle aree caratterizzate dalla piccola impresa si è trovato privo di riferimenti politici dopo la crisi della Dc, partito che aveva garantito integrazione e mediazione politica degli interessi locali in molte province del Nord. Sono così cresciute soprattutto tra $i$ piccoli imprenditori l'insoddisfazione per le politiche nazionali e la domanda di un nuovo tipo di rappresentanza politica, creando il terreno propizio per il progetto della Lega di rappresentare gli interessi delle aree a imprenditorialità diffusa (Diamanti 1996, 19-27).

La scelta del Carroccio a favore della piccola impresa ha assunto una grande importanza anche sul piano simbolico. La piccola impresa, soprattutto a conduzione familiare, bene integrata nella comunità locale, è diventata il sacrario dei valori che caratterizzano i popoli della Padania: laboriosità, intraprendenza, tenacia, schiettezza, capacità di reggere la sfida della mondializzazione dell'economia senza perdere l'attaccamento al territorio e alle sue tradizioni.

La difesa degli interessi della piccola industria ha assunto come terreno privilegiato la protesta fiscale. Il malessere fiscale era cresciuto in Italia nel corso degli anni Ottanta per gli aumenti dei livelli di prelievo, in parallelo al logoramento dei rapporti fra elettori e partiti politici. La critica al sistema fiscale e le richieste di cambiamento attraversavano però gli schieramenti politici: l'attivazione della protesta fiscale appariva poco vantaggiosa, e molto rischiosa per i principali partiti italiani. La Lega ha potuto invece agire da imprenditore politico della protesta fiscale, facilmente inquadrata nella battaglia per la difesa degli interessi delle regioni dell'Italia settentrionale contro gli sprechi e le politiche assistenziali della partitocrazia. Il tema della protesta antitasse, particolarmente sentito dai lavoratori autonomi e dai piccoli produttori, è diventato uno dei terreni principali della lotta contro il governo.

Le campagne leghiste sulle tasse hanno assunto forme molto lontane dal neoliberismo thatcheriano e reaganiano. Il Carroccio ha unito alle richieste di diminuzione dell'imposizione fiscale un chiaro orientamento protezionistico per le comunità locali, e in prospettiva per la Padania. La propaganda leghista non sottolinea tanto l'oppressione del singolo contribuente da parte 
della finanza pubblica quanto le contraddizioni fra le regioni del Nord, che sopportano gran parte del carico fiscale, e quelle meridionali. Gli operai, i piccoli imprenditori, gli artigiani e i commercianti possono ritrovare l'unità nella lotta contro la pressione fiscale dello stato coloniale, che dissipa le risorse delle regioni settentrionali a favore del Mezzogiorno.

I tentativi di mobilitazione diretta sul terreno della protesta fiscale messi in atto dalla Lega hanno avuto finora sempre un esito fallimentare: le proposte di sciopero fiscale sono state seguite da una minoranza trascurabile di contribuenti. Il ruolo di imprenditore della protesta fiscale assunto dalla Lega all'inizio degli anni Novanta ha però avuto effetti importanti sia sul terreno elettorale che sul terreno politico. Dal 1994 l'intero schieramento di centro-destra ha assunto la battaglia per ridurre l'imposizione fiscale come tema caratterizzante. E sono state seguite con grande attenzione iniziative e prese di posizione delle associazioni dei commercianti e dei piccoli imprenditori. Forza Italia e Alleanza nazionale hanno cercato di contendere i consensi leghisti sul terreno della protesta contro le tasse e della rappresentanza degli interessi della piccola impresa. Il Carroccio si è differenziato dal Polo riconducendo la questione fiscale e la potenziale conflittualità fra piccola e grande impresa alla contrapposizione fra gli interessi della Padania e quelli delle altre regioni italiane: solo nel quadro del progetto di superamento dello stato nazionale la pressione fiscale potrà essere ridotta e la piccola e media impresa, i commercianti e gli artigiani potranno trovare una valida tutela.

\section{Una cultura politica di tipo nuovo}

Le posizioni dei partiti italiani erano sempre state sintetizzate, nel discorso dei politici e nel senso comune degli elettori, soprattutto facendo riferimento alla loro collocazione sulla dimensione sinistra/destra. La Lega ha negato rilevanza a questa dimensione, ha ignorato la frattura religiosa e ha contestato, come abbiamo visto, l'interpretazione tradizionale della frattura di classe. Il quadro interpretativo proposto dal Carroccio, molto lontano dalla abituale rappresentazione dei conflitti politici e sociali, è stato inizialmente accolto con disprezzo e ironia dalle forze politiche e dai media. Il nuovo partito è riuscito però in pochi anni a formare e consolidare una originale cultura politi- 
ca che è penetrata profondamente tra gli attivisti e i simpatizzanti leghisti, e viene quotidianamente propagandata dal giornale «la Padania».

Al di là dei contenuti programmatici proposti, a caratterizzare il discorso leghista è stato soprattutto il linguaggio impiegato. Parafrasando la celebre massima di McLuhan, nel caso della Lega si può affermare che «il linguaggio è il messaggio». Il linguaggio proposto dai leghisti evidenzia infatti immediatamente due tipi di rotture: con il linguaggio nazionale ufficiale da una lato, con il linguaggio della classe politica italiana dall'altro. Le rotture sul piano del linguaggio sono state rese più evidenti dalla scelta dei canali di comunicazione. Mentre i partiti tendono a comunicare con il pubblico sempre più attraverso la televisione e la grande stampa, la Lega ha privilegiato a lungo le forme di comunicazione più povere e tradizionali, basate sul contatto diretto con i cittadini. I successi elettorali e la novità e trasgressività dalle iniziative leghiste hanno d'altra parte garantito al Carroccio un accesso significativo al sistema dei media per diversi anni.

La comunicazione leghista ha operato una costante semplificazione dei conflitti politici e sociali, mettendo in evidenza lo scontro decisivo fra i popoli della Padania da una parte, e tutti i possibili nemici (Roma, il Sud, i partiti romani, i fascisti, gli immigrati extracomunitari, la grande impresa, le multinazionali, ecc.) dall'altra. Abbandonata la dicotomia sinistra/destra, la Lega ha cercato di fare emergere soprattutto due nuove linee di conflittualità: una orizzontale, lungo la linee di potenziale frattura fra i diversi popoli e territori; una verticale, che contrappone chi sta in alto e chi sta in basso nelle diverse gerarchie di stratificazione sociale.

La polarizzazione orizzontale contrappone il Nord al Sud, quella verticali la gente comune a tutti i tipi di élite. La conflittualità verticale divide anche il Nord. La gente comune della Padania, la piccola impresa e i suoi lavoratori si contrappongono alla grande industria, alla grande finanza, ai grandi mezzi di comunicazione di massa, sempre presentati nel discorso leghista come alleati del potere politico romano.

Questa concezione dei conflitti politici e sociali è diventata patrimonio degli elettori leghisti, che si differenziano così da tutti gli altri. Grande importanza è attribuita alla frattura Nord/ Sud e alla contrapposizione fra italiani ed extracomunitari. Meno importante è ritenuta la frattura di classe e ancora più ri- 
TAB. 8. Conflitti potenzialmente più importanti" per intenzione di voto

\begin{tabular}{lcccccc}
\hline & \multicolumn{6}{c}{ Elettori } \\
\cline { 2 - 7 } & Lega & An & FI & Ppi & Pds & Nord Italia \\
\hline Nord/Sud & 50,1 & 37,0 & 31,4 & 43,4 & 35,3 & 35,0 \\
Italiani/extracomunitari & 49,2 & 57,2 & 44,1 & 33,5 & 36,3 & 41,8 \\
Lavoratori/imprenditori & 37,5 & 37,9 & 37,8 & 42,7 & 45,1 & 38,1 \\
Destra/sinistra & 22,0 & 26,2 & 27,9 & 38,0 & 30,7 & 23,3 \\
Fascismo/antifascismo & 17,6 & 12,1 & 13,6 & 26,9 & 29,5 & 16,6 \\
Cattolici/laici & 15,2 & 14,1 & 10,2 & 21,1 & 11,0 & 11,7 \\
N. & 166 & 100 & 183 & 67 & 176 & 1200 \\
\hline
\end{tabular}

* L'importanza dei conflitti è stata rilevata con una scala di punteggi fra 1 e 10 . Sono stati considerati rilevanti $\mathrm{i}$ conflitti a cui è stato attribuito un valore non inferiore a 8 .

Fonte: Sondaggio Abacus - Regioni settentrionali (1997).

$\mathrm{TAB}$. 9. Intenzioni di voto per la Lega in diverse aree politiche

\begin{tabular}{|c|c|c|c|c|c|c|c|c|}
\hline & \multicolumn{2}{|c|}{1991} & \multicolumn{2}{|c|}{1994} & \multicolumn{2}{|c|}{1996} & \multicolumn{2}{|c|}{1998} \\
\hline & Voti \% & $\mathrm{N}$ & Voti \% & $\mathrm{N}$ & Voti \% & $\mathrm{N}$ & Voti \% & $\mathrm{N}$ \\
\hline Sinistra & 6,7 & 165 & 3,3 & 874 & 6,0 & 253 & 5,5 & 319 \\
\hline Centro-sinistra & 11,2 & 294 & 7,5 & 306 & 16,1 & 416 & 9,6 & 631 \\
\hline Centro & 14,1 & 404 & 13,8 & 682 & 28,7 & 485 & 29,4 & 833 \\
\hline Centro-destra & 22,5 & 142 & 32,6 & 604 & 18,2 & 364 & 21,5 & 543 \\
\hline Destra & 22,2 & 27 & 28,1 & 506 & 8,1 & 174 & 17,4 & 180 \\
\hline Non collocati & 20,4 & 108 & 23,0 & 261 & 27,9 & 408 & 63,9 & 278 \\
\hline Totale Nord & 14,1 & 1.140 & 16,9 & 3.233 & 19,8 & 2.099 & 22,0 & 2.784 \\
\hline
\end{tabular}
nali.

Fonte: Sondaggi Eurisko (1991) e Abacus (1994; 1996; 1998) - Regioni settentrio-

dotta è la rilevanza attribuita alle contrapposizioni fra destra e sinistra, fra fascismo e antifascismo, e fra cattolici e laici (tab. 8).

La tendenza degli elettori leghisti a rifiutare la tradizionale concezione del conflitto politico si può rilevare anche dalla collocazione personale rispetto alla dimensione destra-sinistra. Fino al 1994 la penetrazione elettorale della Lega era stata più elevata tra gli elettori che si collocavano su posizioni di centro-destra e di destra (tab. 9). Dopo la rottura con il Polo, le probabilità di voto leghista sono cresciute fra gli elettori di centro e, soprattutto nell'ultima fase, fra le persone che rifiutano una collocazione 
sulla dimensione destra-sinistra. La collocazione di molti elettori leghisti al centro non è tanto indicatrice di posizioni moderate, quanto di estraneità a questa dimensione. Questa posizione, da sempre rivendicata dai dirigenti del Carroccio, sembra diventare una caratteristica distintiva dell'elettorato leghista.

La Lega ha cercato di definire la sua identità politica andando al di là dei confini del nostro sistema politico facendo riferimento ad attori e processi che operano a livello internazionale. In positivo, sono stati assunti come referenti ideali i movimenti indipendentisti europei e di tutto il mondo. In negativo, il Carroccio ha accentuato la propria diversità rispetto a tutte le altre forze politiche italiane opponendosi al processo di globalizzazione e alle forme attuali dell'integrazione europea. Le istituzioni e i progetti di integrazione sovranazionali sono considerati funzionali al dominio delle élites finanziarie mondiali

La Lega sottolinea in ogni occasione la contraddizione fra l'Europa dei popoli e l'Unione economica e monetaria attuale, ritenuta espressione degli interessi delle multinazionali e delle banche. Votando contro l'Euro al parlamento europeo, il Carroccio ha cercato di estendere a Bruxelles «verticistica e lontana» la tradizionale lotta contro il centralismo romano. E cambiata di conseguenza la configurazione dei nemici della Padania: i pericoli non provengono solo dal Sud, ma anche dal cuore dell'Europa. Il Carroccio si propone così di diventare il principale imprenditore politico dell'opposizione all'Unione, pronto a raccogliere e ad amplificare i disagi, le delusioni e i problemi che emergeranno nel processo di integrazione europea. La Lega si ricollega idealmente a tutte le posizioni euroscettiche presenti nel vecchio continente, andando contro corrente rispetto a opinioni che sono maggioritarie anche nell'elettorato leghista, con la convinzione di ampliare il proprio consenso su questo terreno a medio termine.

La propaganda leghista enfatizza d'altra parte anche le preoccupazioni popolari per le ripercussioni locali dei processi di internazionalizzazione dei mercati, della finanza e della circolazione della forza lavoro: «Con la globalizzazione si finisce per assumere l'ideologia mondialista, con una rete di interconnessioni e interdipendenze economiche che annullano ogni possibilità di autonomia e indipendenza economica. E quindi politica e culturale sia dei singoli stati che dei popoli» ${ }^{13}$. Contro questi

13 Intervista a Bossi su «la Padania» del 30 dicembre 1998. 
processi, il Carroccio propone la mobilitazione contro l'immigrazione e l'autonomia dell'Italia del Nord per garantire la priorità di accesso al lavoro e ai benefici dello stato sociale per i cittadini padani.

\section{Gli atteggiamenti degli elettori leghisti}

Nel corso degli anni Novanta gli elettori della Lega hanno accentuato alcuni tratti peculiari, differenziandosi dagli elettori dei due principali schieramenti politici. Per mettere in luce questa tendenza, abbiamo confrontato gli atteggiamenti di chi ha votato per il Carroccio con quelli degli elettori residenti nelle regioni del Nord che votano per i principali partiti italiani: il Pds, il Ppi, An e Forza Italia. Abbiamo utilizzato una batteria di indicatori per rilevare un complesso di atteggiamenti: l'autonomismo, il familismo, l'etno-centrismo, la domanda di autorità, il neo-liberismo, il protezionismo e l'operaismo.

È possibile così individuare un gruppo di orientamenti che distinguono l'elettorato leghista dagli altri elettori dell'Italia settentrionale (tab. 10). Chi vota per il partito di Bossi è naturalmente favorevole a rivendicare l'autonomia per le regioni dell'Italia settentrionale. Ma il nordismo si unisce e si integra con una complesso di atteggiamenti ben definito. Tra gli elettori del Carroccio il familismo e l'etno-centrismo hanno una vastissima adesione, superiore al livello che si registra fra gli elettori del Polo e molto superiore a quello degli elettori dell'Ulivo ${ }^{14}$. La domanda di autonomia territoriale proposta dalla Lega ha recuperato e reinterpretato il tradizionale «familismo amorale», storicamente molto diffuso nel nostro paese ${ }^{15}$. Negli anni Novanta il particolarismo si intreccia facilmente all'ostilità verso gli stra-

14 Gli orientamenti familisti sono stati rilevati in base al livello di consenso espresso per la proposizione: «La responsabilità primaria è verso la propria famiglia e non verso la collettività». Gli orientamenti etno-centristi sono stati rilevati in base al livello di consenso espresso per diverse proposizioni che esprimono ostilità nei confronti degli immigrati extracomunitari («Troppi immigrati disturbano»; «Gli immigrati sfruttano il nostro welfare»; «Gli immigrati aumentano la disoccupazione»; «Gli immigrati sono una causa di delinquenza»).

15 Il concetto di «familismo amorale» è stato proposto da Banfield per indicare la tendenza degli abitanti di un paese del Sud oggetto della ricerca a massimizzare i vantaggi materiali e immediati della famiglia e l'incapacità a impegnarsi «per il bene di tutti, cioè per qualsiasi finalità che trascenda l'immediato interesse materiale del nucleo familiare» (Banfield 1958, 10). 
TAB. 10. Atteggiamenti su questioni politiche e sociali per intenzione di voto

\begin{tabular}{lcccccc}
\hline & \multicolumn{6}{c}{ Elettori } \\
\cline { 2 - 7 } & Lega & An & FI & Ppi & Pds & Nord Italia \\
\hline Autonomismo & 88,8 & 73,3 & 68,7 & 64,1 & 60,4 & 69,0 \\
Familismo & 80,9 & 79,3 & 76,9 & 62,1 & 62,6 & 71,5 \\
Etno-centrismo & 77,4 & 73,4 & 66,5 & 49,9 & 55,1 & 63,6 \\
Fiducia sociale & 44,4 & 49,0 & 48,6 & 58,6 & 51,3 & 48,5 \\
Domanda di autorità & 66,5 & 70,2 & 64,9 & 38,7 & 43,0 & 54,2 \\
Neo-liberismo & 61,8 & 67,0 & 64,9 & 50,6 & 49,0 & 55,9 \\
Protezionismo & 59,5 & 55,9 & 58,5 & 49,5 & 57,8 & 57,4 \\
Operaismo & 53,7 & 36,3 & 42,8 & 48,9 & 61,6 & 51,3 \\
N. & 400 & 213 & 441 & 201 & 585 & 2.784 \\
\hline
\end{tabular}

Fonte: Sondaggio Abacus - Regioni settentrionali (1998).

nieri, che rappresenta un'altra faccia della tendenza alla chiusura sociale. Per completare il quadro, possiamo osservare che tra gli elettori del Carroccio la fiducia sociale - le aspettative positive nei confronti di possibili interlocutori nella vita sociale - tocca il livello minimo ${ }^{16}$. E questo complesso di atteggiamenti, e non la semplice domanda di autonomia del Nord, che caratterizza il tipico elettore della Lega.

Due tratti accomunano invece gli elettori del Carroccio e del Polo, differenziandoli nettamente da quelli dei partiti dell'Ulivo: la domanda di autorità - la richiesta di leader forti e l'esigenza di inasprire le pene per chi infrange l'ordine sociale e gli orientamenti neo-liberisti ${ }^{17}$.

Esistono però anche differenze importanti fra gli elettori leghisti e quelli dei partiti del centro-destra, soprattutto sulle questioni economiche e sociali. Il consenso ai classici temi del neo-liberismo si accompagna tra gli elettori della Lega a una domanda di protezione sociale e ad orientamenti di tipo operai-

16 Abbiamo rilevato la fiducia sociale in base al consenso per le affermazioni: «Gran parte della gente è degna di fiducia» e «Ritengo che gli altri siano, nei miei confronti, sempre corretti».

$17 \mathrm{La}$ domanda di autorità è stata rilevata in base al consenso per le affermazioni: «Occorre la pena di morte per i reati più gravi» e «Occorrono persone forti per decisioni importanti». Gli orientamenti neo-liberisti sono stati rilevati in base al consenso per le affermazioni: «Più libertà per l'iniziativa privata e gli affari» e «I servizi sociali dovrebbero essere pagati da chi li usa». 
TAB. 11. Opinioni sulla politica per intenzione di voto

\begin{tabular}{lcccccc}
\hline & \multicolumn{6}{c}{ Elettori } \\
\cline { 2 - 7 } & Lega & An & FI & Ppi & Pds & Nord Italia \\
\hline $\begin{array}{l}\text { La politica mi interessa } \\
\text { La politica è noiosa, starle }\end{array}$ & 18,8 & 39,0 & 36,1 & 41,2 & 51,6 & 37,5 \\
dietro è una perdita di tempo & 49,5 & 34,6 & 43,8 & 27,1 & 25,8 & 38,0 \\
$\begin{array}{l}\text { La gente comune non influenza } \\
\text { il governo }\end{array}$ & 61,4 & 51,4 & 55,4 & 47,2 & 39,0 & 51,2 \\
N. & 229 & 172 & 341 & 430 & 228 & 2.107 \\
\hline
\end{tabular}

Fonte: Sondaggio Abacus - Regioni settentrionali (1996).

$\mathrm{sta}^{18}$. Queste posizioni appaiono accentuate dal mutamento del profilo sociale del voto leghista degli ultimi due anni (tab. 2). Il Carroccio gestisce la domanda di protezione sociale per i lavoratori e per i ceti popolari delle regioni dell'Italia settentrionale rifiutando gli obiettivi e la mediazione delle organizzazioni sindacali confederali. La Lega ha fondato proprie organizzazioni sindacali che rivendicano per $\mathrm{i}$ lavoratori delle regioni del Nord salari più elevati e un accesso privilegiato al lavoro e alla previdenza sociale.

In generale gli elettori leghisti si differenziano da tutti gli altri per un rapporto molto critico nei confronti della politica. Chi vota per il Carroccio dimostra un interesse per la politica molto ridotto, nettamente inferiore a quello degli elettori del Polo e dell'Ulivo (tab. 11). Lo scarso interesse si accompagna a sentimenti negativi rispetto ai contenuti propri della vita politica («la politica è sempre uguale e starle dietro è una noia») e a sentimenti di impotenza nei confronti dell'esercizio del potere politico, diffusi tra elettori leghisti molto più che tra gli elettori degli altri partiti.

E ai livelli minimi, tra gli elettori della Lega, la fiducia nei confronti dei partiti politici e delle istituzioni democratiche rappresentative (tab. 12). Rispetto alla Regione e al Comune la fi-

18 Gli orientamenti per la protezione sociale sono stati rilevati sulla base del consenso all'affermazione: «I servizi sociali dovrebbero essere gratis per tutti». Gli orientamenti operaisti sono stati rilevati tenendo conto del consenso per l'affermazione: «C'è bisogno di più potere ai lavoratori», e del dissenso per l'affermazione: «Occorrerebbero meno scioperi». 
TAB. 12. Fiducia nei partiti politici e nelle istituzioni rappresentative, per intenzione di voto

\begin{tabular}{lcccccc}
\hline & \multicolumn{6}{c}{ Elettori } \\
\cline { 2 - 7 } & Lega & An & FI & Ppi & Pds & Nord Italia \\
\hline Fiducia in: & & & & & & \\
Partiti politici & 19,9 & 20,3 & 20,4 & 40,8 & 49,1 & 30,4 \\
Parlamento & 30,2 & 27,9 & 31,3 & 70,2 & 72,0 & 47,7 \\
Comune & 56,8 & 51,2 & 52,0 & 77,6 & 76,4 & 63,7 \\
Regione & 56,3 & 48,8 & 54,0 & 75,9 & 71,5 & 61,5 \\
N. & 229 & 172 & 341 & 430 & 228 & 2.107 \\
\hline
\end{tabular}

Fonte: Sondaggio Abacus - Regioni settentrionali (1996).

ducia degli elettori leghisti cresce, ma si mantiene al di sotto di quella espressa in media nelle regioni del Nord. Anche per le istituzioni rappresentative che possono essere più vicine alle esigenze dei cittadini e alle comunità locali la diffidenza resta elevata.

Nell'ottica leghista, la politica viene ad assumere connotati fortemente negativi, coincide con la partitocrazia e con l'uso della politica a fini personali. Il Carroccio aveva conquistato un vasto consenso elettorale nei primi anni Novanta con la lotta intransigente contro questi fenomeni. La scarsa fiducia nella politica resta un tratto distintivo degli elettori leghisti, alimentata anche dalla loro tendenza generalizzata alla diffidenza sociale.

\section{Un nuovo partito di massa?}

Conquistando consensi nelle aree sociali più critiche rispetto alla politica, la Lega Nord è riuscita in pochi anni a costruire uno dei più importanti attori politici italiani. In una fase di crisi della partecipazione e di declino dei partiti di massa, il nuovo soggetto politico è riuscito a promuovere nuova militanza e a creare una forte organizzazione, dotata di una rete di sezioni diffusa sul territorio e rafforzata da un complesso di organizzazioni fiancheggiatrici.

Quale modello organizzativo ha assunto il nuovo partito? La fisionomia organizzativa è condizionata in generale da tre questioni cruciali: a) la fonte di legittimazione (interna o esterna al partito); b) il modo in cui è stata costruita l'organizzazione (per penetrazione o per diffusione); c) l'esistenza o no di una 
leadership carismatica (Panebianco 1982, 105-110). La Lega non ha avuto alcun tipo di legittimazione esterna da parte di movimenti e istituzioni preesistenti, ma ha conquistato l'adesione dei militanti e degli elettori con il lungo lavoro di proselitismo svolto direttamente dalle piccole minoranze dei fondatori. La costruzione della Lega Nord si è realizzata con la federazione delle originarie leghe autonomiste, seguendo apparentemente un processo di sviluppo organizzativo basato sul modello della diffusione territoriale. In realtà, le singole leghe in Veneto, Lombardia e Piemonte si sono costituite per penetrazione sul territorio a partire da un centro che, in ciascuna regione, ha diretto e controllato attentamente il reclutamento dei militanti e la presentazione delle liste nelle diverse tornate elettorali. La creazione della Lega Nord si è realizzata con l'integrazione delle leghe regionali nell'ambito del modello organizzativo creato dalla Lega Lombarda, che ha espresso il gruppo dirigente del nuovo soggetto politico, emarginando progressivamente $i$ fondatori delle altre leghe.

Il modello organizzativo della Lega ha riprodotto i tratti tipici di tutti i partiti fondati su una leadership carismatica. L'accesso a tutti i ruoli di potere nell'organizzazione è diventato sempre più esclusivamente dipendente dall'appoggio e dalla fiducia del leader. Si è formato un gruppo dirigente unito dalla fedeltà a Bossi che rappresenta l'unico punto di riferimento e di aggregazione. I militanti e i sostenitori sono scarsamente disponibili a iniziative scissionistiche, che risultano sempre marginali e destinate alla sconfitta. Il processo di istituzionalizzazione del partito è stato spesso ostacolato da Bossi. Gli uffici e le responsabilità a livello centrale vengono spesso «rivoluzionati» dal segretario, per impedire che si cristallizzi un apparato burocratico stabile. La Lega tende a presentarsi come movimento anti-partito, che si contrappone a tutti i partiti esistenti. L'apparato di funzionari è tuttora ridotto al minimo sia a livello centrale che provinciale. Gran parte dell'attività politica è costituita dall'impegno volontario dei militanti.

La sovrapposizione fra l'identità dell'organizzazione e il leader è progressivamente cresciuta nel corso dello sviluppo della Lega. Bossi appare a militanti e sostenitori come l'incarnazione del volere del popolo, di cui recupera ed esprime i valori autentici in opposizione alle élites politiche, economiche e culturali dominanti. Il rapporto fra il segretario e i militanti di base riproduce il rapporto che dovrebbe stabilirsi fra leader e popolo come è immaginato e idealizzato dai movimenti populisti. 
La struttura organizzativa della Lega Nord, formalmente basata su criteri federativi, ha assunto una fisionomia sempre più accentrata, rafforzata dallo stile di direzione di Bossi. I dirigenti regionali hanno un rilievo sostanzialmente marginale: le decisioni locali vengono sempre ispirate e attentamente vagliate da un ristretto centro politico. Bossi detiene la quasi totalità del potere politico nell'ambito del movimento: lo stesso Consiglio Federale ha un ruolo limitato di fronte al leader. Altri dirigenti che hanno cercato in qualche misura di autonomizzarsi dal segretario hanno finito per allontanarsi dall'organizzazione, o sono stati espulsi.

Il consolidamento dell'assetto organizzativo della Lega è stato favorito, nei primi anni novanta, da due condizioni esterne: la stabilità delle aree di espansione, ove il movimento si è progressivamente insediato, e l'ostilità proveniente dall'esterno, molto superiore al normale antagonismo delle competizioni elettorali. L'ostilità percepita nell'ambiente esterno ha favorito una forte coesione interna. Gli attivisti della Lega sono caratterizzati da alcuni tratti peculiari: una forte carica antagonista e protestataria; l'estraneità rispetto alle tradizioni politiche italiane cattoliche, socialiste e comuniste; l'ambizione a porsi come portavoce del senso comune popolare, in particolare nella espressione di ostilità verso il ceto politico e intellettuale nazionale e verso gli immigrati.

Lo stile di militanza leghista mantiene tuttora un profilo tipico: a) l'impegno personale è un dovere, non una semplice possibilità; b) la partecipazione è prevalentemente esecutiva, e scarsamente propositiva o decisionale; $c$ ) si valorizza molto l'impegno manuale, e poco quello intellettuale. Per la carriera politica titolo di studio e ruolo sociale del militante non contano. Il principio che vale è l'anzianità come garanzia di fedeltà alla causa.

Per l'impegno richiesto, la Lega ha distribuito ai militanti sia incentivi collettivi (incentivi legati all'identità, e al perseguimento dei valori condivisi) che incentivi selettivi (vantaggi individuali materiali o di status). La Lega ha potuto distribuire molti incentivi di identità ai militanti e agli iscritti, perché ha mantenuto, sostanzialmente indisturbata, il monopolio dei fini che professa. La distribuzione degli incentivi selettivi di potere e status dipende sia dall'organizzazione, che offre la possibilità di fare carriera molto velocemente, sia dal leader, che chiama direttamente alcuni militanti per compiti importanti. Le afferma- 
zioni elettorali e gli sviluppi organizzativi hanno garantito a molti militanti incentivi materiali e di status. Per i militanti, il grado di sostituibilità del movimento con altre formazioni politiche è molto limitato, perché la Lega offre incentivi, sia collettivi che selettivi, che nessun'altra organizzazione può offrire.

La Lega ha perseguito il progetto tipico dei grandi partiti di massa: la costruzione di organizzazioni fiancheggiatrici per raccogliere adesione e consenso popolare in molteplici sfere della vita sociale. Il Carroccio ha promosso, fin dalle origini, sindacati, associazioni di categoria, gruppi culturali, ricreativi e sportivi. Le associazioni leghiste hanno col partito un rapporto più stretto e minore autonomia politica e organizzativa rispetto alle organizzazioni fiancheggiatrici dei tradizionali partiti di massa. La Lega non solo ha promosso direttamente la formazione delle associazioni, ma ne governa l'evoluzione sulla base delle proprie strategie politiche. Le sedi centrali e periferiche delle organizzazioni leghiste si trovano nelle sedi centrali e in alcune sedi periferiche del partito.

\section{La trasformazione del mercato elettorale nelle regioni del Nord}

La configurazione del mercato elettorale nelle regioni dell'Italia settentrionale era rimasta sostanzialmente stabile dal dopoguerra agli anni Ottanta. Si potevano identificare tre zone nettamente differenziate ${ }^{19}$. Due aree registravano il tradizionale predomino di una subcultura politica: la subcultura «bianca» nelle regioni del Triveneto e in alcune province lombarde, e la subcultura «rossa» dominante soprattutto in Emilia-Romagna. In queste aree predominava il voto di appartenenza, e si registrava una mobilità elettorale molto limitata. La terza area - la «zona industriale» che gravitava sul triangolo Milano, Torino e Genova - era invece identificata più per il ruolo svolto dalla grande industria nella struttura sociale che per il predominio di una particolare cultura politica. I partiti di governo disponevano della maggioranza dei voti nella zona industriale. Ma l'oppo-

19 La suddivisione del Nord in tre aree omogenee dal punto di vista della tradizione politica è stata elaborata negli anni Sessanta dai ricercatori dell'Istituto Cattaneo (Galli et al. 1968). Questa suddivisione è stata accettata da quasi tutti i ricercatori, anche se sono stati in certi casi parzialmente modificati i confini delle tre zone (Cartocci 1987; Corbetta, Parisi e Schadee 1988, 506). 
sizione di sinistra era in grado di contendere il primato elettorale in molte situazioni e poteva contare sul sostegno del movimento operaio organizzato per molte battaglie politiche. Nella zona industriale al voto di appartenenza si affiancava in misura significativa il voto di opinione, che rendeva più elevato il livello di mobilità elettorale, soprattutto nelle grandi città.

L'ondata leghista dei primi anni Novanta ha profondamente trasformato la geografia elettorale nelle regioni del Nord. Partita dalla Lombardia, ha poi investito completamente la zona bianca, arrivando a toccare alcune province della zona rossa. Originata nei piccoli centri di provincia, è arrivata nel 1993 anche a coinvolgere le principali metropoli del Nord. La decisione di votare per la Lega, anche per una sola volta, ha significato spesso la rottura con i precedenti riferimenti politici. Solo una parte di queste scelte ha originato una duratura fedeltà elettorale al Carroccio. Più in generale, si è creata una temporanea situazione di grande mobilità elettorale a causa della crisi dei partiti di governo. Forza Italia e, in misura minore, Alleanza nazionale hanno raccolto nel 1994 molti consensi di ex elettori del pentapartito che, in molti casi, avevano già sperimentato il voto per la Lega. Il partito di Bossi ha perso voti soprattutto nelle aree metropolitane, e ha mantenuto e rafforzato il proprio originario bacino elettorale in molte province della Lombardia, del Veneto, del Friuli e del Piemonte.

La nuova geografia elettorale che si è creata nell'Italia settentrionale si può studiare sulla base dei risultati delle elezioni del 1996 per la Camera. Abbiamo analizzato con la tecnica della cluster analysis i voti raccolti nei collegi del Nord dai sei partiti che hanno ottenuto più voti per la parte proporzionale. Abbiamo escluso dall'analisi i collegi della Valle d'Aosta e del Sud Tirolo, che presentano caratteristiche particolari per il ruolo dominante svolto dai partiti etno-regionalisti.

Si possono individuare quattro tipi di collegio, che restano sostanzialmente stabili a fronte di ripetute analisi ${ }^{20}$ (tab. 13).

20 Le tecniche di cluster analysis producono in generale una pluralità di soluzioni, che possono presentare notevoli differenze a seconda dei criteri e dei metodi adottati (Ricolfi 1989). Per ridurre l'arbitrarietà delle scelte, abbiamo condotto diversi test per verificare la stabilità della soluzione (Biorcio e Pagani 1997, 228). È stata scelta la soluzione a quattro gruppi perché un aumento del loro numero produceva un aumento molto piccolo della varianza complessiva spiegata (da $52 \%$ al $53 \%$ ). La configurazione del mercato elettorale emersa dalla nostra analisi è congruente con quella delineata da Diamanti $(1996,85)$, che ha utilizzato un diverso insieme di variabili e una differente tecnica di analisi. 
TAB. 13. Tipi di collegio elettorale per regione (Nord-Camera 1996, quota proporzionale)

\begin{tabular}{lccccc}
\hline & \multicolumn{5}{c}{ Tipo di collegio } \\
\cline { 2 - 6 } & $\begin{array}{c}\text { Prevalenza } \\
\text { Lega }\end{array}$ & $\begin{array}{c}\text { Prevalenza } \\
\text { Sinistra }\end{array}$ & $\begin{array}{c}\text { Competizione } \\
\text { Polo-Ulivo }\end{array}$ & $\begin{array}{c}\text { Competizione } \\
\text { Tripolare }\end{array}$ & Totale \\
\hline Piemonte & 1 & 0 & 24 & 11 & 36 \\
Lombardia & 18 & 1 & 30 & 25 & 74 \\
Trentino & 0 & 0 & 2 & 2 & 4 \\
Veneto & 14 & 0 & 9 & 14 & 37 \\
Friuli & 3 & 0 & 4 & 3 & 10 \\
Liguria & 0 & 7 & 7 & 0 & 14 \\
Emilia & 0 & 28 & 4 & 0 & 32 \\
Totale & 36 & 36 & 80 & 55 & 207 \\
\hline
\end{tabular}

ТАВ. 14. Scelte di voto per tipo di collegio elettorale (Nord-Camera 1996, quota proporzionale)

\begin{tabular}{|c|c|c|c|c|c|}
\hline \multirow[b]{2}{*}{ Voto } & \multicolumn{5}{|c|}{ Tipo di collegio } \\
\hline & $\begin{array}{l}\text { Prevalenza } \\
\text { Lega }\end{array}$ & $\begin{array}{l}\text { Prevalenza } \\
\text { Sinistra }\end{array}$ & $\begin{array}{c}\text { Competizione } \\
\text { Polo-Ulivo }\end{array}$ & $\begin{array}{c}\text { Competizione } \\
\text { Tripolare }\end{array}$ & Totale \\
\hline Lega & 39,8 & 6,6 & 14,6 & 27,1 & 20,5 \\
\hline Forza Italia & 17,5 & 15,0 & 23,1 & 21,4 & 20,1 \\
\hline Alleanza Nazionale & 7,8 & 11,3 & 12,7 & 10,5 & 11,1 \\
\hline Ccd-Cdu & 5,7 & 4,5 & 4,3 & 5,4 & 4,8 \\
\hline Lista Dini & 4,0 & 4,0 & 4,4 & 3,7 & 4,2 \\
\hline Ppi & 7,7 & 7,6 & 6,3 & 7,4 & 7,3 \\
\hline Verdi & 2,0 & 2,6 & 2,8 & 2,6 & 2,6 \\
\hline Pds & 8,6 & 36,7 & 18,9 & 12,8 & 18,6 \\
\hline Rif. Comunista & 4,3 & 8,9 & 9,5 & 6,0 & 7,5 \\
\hline N. collegi & 36 & 36 & 80 & 55 & 212 \\
\hline
\end{tabular}

Per caratterizzare le quattro aree, abbiamo calcolato le percentuali di voti raccolti dai sei partiti in ciascuna di esse (tab. 14).

I primi due tipi presentano un profilo molto preciso, segnato da un orientamento politico nettamente dominate. Trentasei collegi sono caratterizzati da una forte presenza della Lega, che vi raccoglie in media il $40 \%$ dei consensi. I partiti di sinistra sono deboli. Il Polo appare invece in grado di contendere il primato alla Lega in diverse situazioni. Questo primo tipo di collegio è diffuso soprattutto in Lombardia e Veneto, ma comprende anche collegi piemontesi e friulani. Non ne fa parte alcun collegio di area metropolitana. Nel secondo tipo di collegi si re- 
gistra la netta prevalenza dei partiti di sinistra eredi del Pci, che raccolgono in media il $46 \%$ dei voti. Questo tipo comprende quasi tutti i collegi dell'Emilia-Romagna, metà dei collegi liguri e un collegio lombardo, e rispecchia in gran parte la configurazione della zona rossa, che ha resistito molto meglio della zona bianca all'ondata elettorale leghista.

I due altri tipi di collegio presentano una struttura politica più complessa e più aperta alla competizione elettorale ${ }^{21}$. In 80 collegi la competizione è fra Polo e Ulivo, con una maggioranza relativa di Forza Italia. La Lega raccoglie consensi nettamente al di sotto della media e non è in grado di modificare la struttura bipolare della competizione. Questo tipo di collegio è diffuso in tutte le regioni, soprattutto in Piemonte e Lombardia, e comprende tutti i collegi metropolitani di Milano, Torino, Venezia e Trieste. Nei 55 collegi del quarto tipo il Carroccio è invece il partito più votato $(27 \%)$, ed è spesso in grado di competere con i due principali schieramenti politici nazionali. La competizione risulta spesso tripolare (Polo, Ulivo, Lega). Si tratta di collegi di piccoli e medi centri del Piemonte, della Lombardia e del Veneto.

La Democrazia cristiana e i partiti alleati di governo avevano sempre avuto, dal dopoguerra agli anni Ottanta, una larga maggioranza di consensi nelle regioni settentrionali. Il voto del Nord ha per molto tempo offerto un importante sostegno al governo nazionale. Negli anni Novanta il voto dei cittadini dell'Italia settentrionale è diventato un fattore di instabilità per il sistema politico italiano. Le tensioni e i problemi che la Lega ha utilizzato per conquistare un vasto consenso si mantengono e non sembrano destinati ad attenuarsi nel prossimo futuro. Continua a sussistere, anche dopo il ricambio di parte della classe politica, scarsa sintonia fra cittadini e partiti politici. Cresce l'astensionismo elettorale. Si ripropone e si acutizza la frattura Nord-Sud per i problemi sollevati dall'integrazione europea e dalla globalizzazione dell'economia. In questo quadro, può apparire vantaggioso e ragionevole per le regioni dell'Italia del Nord gestire in modo autonomo il proprio destino, lasciando cadere ogni dovere di solidarietà nazionale. A questa prospettiva si dimostrano particolarmente sensibili gli strati popolari a

21 Mentre i primi due tipi risultano stabili di fronte a ripetute analisi, il terzo e il quarto appaiono meno stabili per la presenza di alcuni collegi di «confine» che possono essere assegnati indifferentemente all'una o all'altra tipologia. 
cui soprattutto si rivolge il Carroccio: lavoratori e imprenditori delle piccole imprese, piccoli artigiani e commercianti, e in generale $\mathrm{i}$ settori meno istruiti della popolazione che risiedono nelle zone periferiche dell'Italia settentrionale.

La presenza e la forza elettorale mantenute dalla Lega impediscono sia al centro-destra che al centro-sinistra la conquista della maggioranza dei voti del Nord. Il Carroccio cerca di sfruttare tutti gli spazi di azione politica creati dalla lunga transizione italiana per impedire un riassetto bipolare del sistema: «Il bipolarismo non esiste, se c'è un bipartitismo, è quello delle differenze reali fra Nord e Sud» ${ }^{22}$. Le tensioni, i problemi e le domande che emergono dall'Italia settentrionale sono d'altra parte troppo eterogenei per consentire alla Lega di estendere la sua egemonia politica al di là delle roccaforti tradizionali. Caduta la speranza di unificare la Padania sotto le proprie bandiere, la dirigenza leghista sembra ridimensionare le attese alla possibilità che i propri voti diventino indispensabili per la formazione di un futuro governo nazionale.

\section{Riferimenti bibliografici}

Anastasia, B. e G. Corò, (1996), Evoluzione di un'economia regionale. Il Nordest dopo il successo, Portogruaro, Ediciclo.

Banfield, E. (1958), The Moral Basis of a Backward Society, Glencoe, Ill., The Free Press, trad. it. Le basi morali di una socità arretrata, Bologna, Il Mulino, 1976.

Bagnasco, A. (1997), Cambiamento sociale in tempo di cambiamento politico, in N. Negri e L. Sciolla (a cura di), Il paese dei paradossi, Roma, Nis, pp. 147-192.

Becchi, A. (1995), La questione meridionale, in Pasquino (1995), pp. 495-506.

Biorcio, R. (1991), La Lega Lombarda come attore politico: dal federalismo al populismo regionalista, in Mannheimer (1991), pp. 34-82.

- (1997), La Padania promessa. La storia, le idee e la logica d'azione della Lega Nord, Milano, Il Saggiatore.

Biorcio, R. e S. Pagani (1997), Introduzione alla ricerca sociale, Roma, Nis.

Cartocci, R. (1987), Otto risposte a un problema: la divisione dell'Italia in zone politicamente omogenee, in «Polis», n. 3, pp. 481-514.

22 Dichiarazione di Domenico Comino, capogruppo della Lega alla Camera, a «la Repubblica» del 7 marzo 1998. 
Confalonieri, M.A. (1997), Identità, interessi e carisma nei movimenti populisti: la Lega Nord e il Poujadismo, in «Quaderni di Scienza politica», n. 1, pp. 53-85.

Corbetta, P. (1993), La Lega e lo sradicamento del sistema, in «Polis», n. 2, pp. 229-252.

Corbetta, P. e A. Parisi (1994), Ancora due Italie. Sulla natura della diversità meridionale nel referendum del 18 aprile 1993, in «Polis», n. 1, pp. 11-33.

Corbetta, P., A. Parisi e H. Schadee (1988), Elezioni in Italia, Bologna, Il Mulino

De Winter, L. e H. Türsan (1998), Les partis ethno-regionalistes en Europe, in «Revue International de Politique Comparée», n. 1, pp. 151-163.

Diamanti, I. (1996), Il male del Nord, Roma, Donzelli.

- (1997), Nordest: si può crescere senza politica?, in «il Mulino», n. 6, pp. 1061-1073.

Diamanti, I. e P. Segatti (1994), Orgogliosi di essere italiani, in «Limes», n. 4, pp. 37-44.

Diani, M. (1996), Linking Mobilisation Frames and Political Opportunities: Insight from Regional Populism in Italy, in «American Sociological Review», vol. 61, pp. 1053-1069.

Galli, G., V. Capecchi, V. Cioni Polacchini e G. Sivini (1968), Il comportamento elettorale in Italia, Bologna, Il Mulino.

Hobsbawn, E.J. (1983), Introduzione a E.J. Hobsbawn e T. Ranger, The Invention of Tradition, Cambridge, Cambridge University Press, trad. it. L'invenzione della tradizione, Torino, Einaudi, 1987.

Kitschelt, H. (1993), Blending New Right Appeals in a Broader Populist anti-Establishment Strategy: Austria and Italy, in H. Kitschelt e A.J. McGann (a cura di), The Radical Right in Western Europe, Ann Arbor, The University of Michingan Press, pp. 159-201.

Mannheimer, R. (1993) L'elettorato della Lega Nord, in «Polis», n. 2, pp. 253-274.

- (a cura di) (1991), La Lega Lombarda, Milano, Feltrinelli.

Natale, P. (1997), Il disagio del Nord. Malessere sociale e dintorni, in «Orientamenti», n. 9-10, pp. 11-28.

Pacini, M. (1992), Introduzione a AA.VV, La Padania: una regione italiana in Europa, Torino, Edizioni della Fondazione Giovanni Agnelli.

Panebianco, A. (1982), Modelli di partito, Bologna, Il Mulino.

Pasquino, G. (a cura di) (1995), La politica italiana. Dizionario critico 1945-95, Roma-Bari, Laterza.

Ricolfi, L. (1989), Sul mito delle K Italie. Una critica delle tipologie degli italiani, in «Polis», n. 3, pp. 445-478.

- (1995) La lega, in Pasquino (1995), pp. 262-271.

Rusconi, G.E. (1993), Se cessiamo di essere una nazione, Bologna, Il Mulino. 
Segatti, P. (1995), Una nazione di paesani. Localismo e sentimento nazionale, in A. Parisi e H. Schadee (a cura di), Sulla soglia del cambiamento, Bologna, Il Mulino, pp. 105-138.

Taggart, P.A. (1996), The New Populism and the New Politics, London, Macmillan.

Trigilia, C. (1994), Nord e Sud: se il Belpaese si spezza, in «Limes», n. 4, pp. 81-94.

- (1997), Dinamismo privato e disordine pubblico, in N. Negri e L. Sciolla (a cura di), Il paese dei paradossi, Roma, Nis, pp. 147-192. 